\title{
Analysis and Simulation of the Early Warning Model for Human Resource Management Risk Based on the BP Neural Network
}

\author{
Xue Yan $\mathbb{D}^{1},{ }^{1}$ Xiangwu Deng, ${ }^{2}$ and Shouheng Sun ${ }^{1}$ \\ ${ }^{1}$ School of Economics and Management, University of Science and Technology Beijing, Beijing 100083, China \\ ${ }^{2}$ School of Electronic Information Engineering, Guangdong University of Petrochemical Technology, Maoming, \\ Guangdong 525000, China
}

Correspondence should be addressed to Xue Yan; b20150372@xs.ustb.edu.cn

Received 22 September 2020; Revised 27 October 2020; Accepted 7 November 2020; Published 18 November 2020

Academic Editor: Wei Wang

Copyright $\odot 2020$ Xue Yan et al. This is an open access article distributed under the Creative Commons Attribution License, which permits unrestricted use, distribution, and reproduction in any medium, provided the original work is properly cited.

\begin{abstract}
Human resource management risks are due to the failure of employer organization to use relevant human resources reasonably and can result in tangible or intangible waste of human resources and even risks; therefore, constructing a practical early warning model of human resource management risk is extremely important for early risk prediction. The back propagation (BP) neural network is an information analysis and processing system formed by using the error back propagation algorithm to simulate the neural function and structure of the human brain, which can handle complex and changeable things that do not have an obvious linear relationship between output results and input factors, so as to find the objective connection between the two. Based on the summary and analysis of previous research works, this article expounded the research status and significance of early warning for human resource management risks, elaborated the development background, current status, and future challenges of the BP neural network, introduced the method and principle of the BP neural network's connection weight calculation and learning training, performed the risk inducement analysis, index system establishment, and network node selection of human resource management, constructed an early warning model of human resource management risk based on the BP neural network, conducted the risk warning model training and detection based on the BP neural network, and finally carried out a simulation and its result analysis. The study results show that the early warning model of human resource management risk based on the BP network is effective, and this trained and tested BP network risk warning model can be used to conduct early warning empirical research on human resource risks to prevent human resource risks, ensure enterprise's benign operation, and at the same time play a role in supervision and promotion of market order rectification.
\end{abstract}

\section{Introduction}

Human resources have become an indispensable key resource for the development of enterprises, and enterprises have gradually realized its role and importance and raised it to an unprecedented height. Human resource management is the core support and strong guarantee for the survival and innovation and development of an enterprise, and it is directly related to the realization of the strategic development goals of the enterprise [1]. Human resource management risks are due to the failure of the employer organization to use relevant human resources reasonably, resulting in tangible or intangible waste of human resources, and even risks. The scope of this risk will involve the recruitment, training, performance appraisal, salary, and other key links of human resources [2]. If the risky enterprises in these important links are not handled properly, it will cause immeasurable losses to the company and even cause the company's decline. Due to the inherent special attributes of human resources, the risks of human resource management are more concealed than other business risks and are often overlooked [3]. Therefore, constructing a practical early warning model of human resource management risk is extremely important for early risk prediction, and risk management plays a pivotal role in the entire management system of an enterprise. In the field of risk management, research studies have been conducted for a long time with a very extensive scope, and scholars' research methods are also constantly innovating [4]. 
The artificial neural network is a mathematical model established by simulating the behavioral characteristics of the human brain neural network, which has strong selfadaptive ability and can adjust the internal nodes of the network according to the complex situation of different systems [5]. The back propagation (BP) artificial neural network is an information analysis and processing system that uses the error back propagation algorithm to simulate the neural function and structure of the human brain [6]. Through BP neural network analysis, it can handle complex and changeable things that do not have an obvious linear relationship between output results and input factors, so as to find the objective connection between the two. The BP neural network can generate reasonable output for new inputs during the training process, which greatly reduces the difficulty of risk assessment of corporate marketing channels. In order to avoid human interference and errors, through system identification and control, the optimal algorithm is used to optimize system functions to realize the early warning and control functions of the future development of things [7]. Therefore, the introduction of the BP neural network into the human resource early warning indicator evaluation system can effectively solve the problems of the nonlinear model of early warning indicators and the difficulty of adapting to online timing early warning, improve the efficiency of system early warning, and provide the necessary technical guarantee for the correct early warning of the early warning system [8].

Based on the summary and analysis of previous research works, this article expounded the research status and significance of early warning for human resource management risks, elaborated the development background, current status, and future challenges of the BP neural network, introduced the method and principle of the BP neural network's connection weight calculation and learning training, performed the risk inducement analysis, index system establishment, and network node selection of human resource management, constructed an early warning model of human resource management risk based on the BP neural network, conducted the risk warning model training and detection based on the BP neural network, and finally carried out a simulation and its result analysis. The study results of this paper will provide a reference for further research studies on the analysis and simulation of the early warning model of human resource management risk based on the BP neural network. The detailed chapters are arranged as follows: Section 2 introduces the method and principle of the BP neural network's connection weight calculation and learning training. Section 3 constructs an early warning model of human resource management risk based on the BP neural network. Section 4 conducts the risk warning model training and detection based on the BP neural network. Section 5 carries out a simulation and its result analysis.

\section{Methods and Principles}

2.1. Connection Weight Calculation of the BP Neural Network. The BP neural network's input layer collects external related information and passes the input information to the competition layer [9]. This layer recategorizes and combines the information, finds the rules, and arranges them in order. Among them, the minimum transmission distance between neurons can be expressed as

$$
P(x)=\left|A_{x}-B_{x}\right|=\sqrt{\sum_{x=1}^{n}\left[A_{x}-B_{x}\right]^{2}}
$$

where $n$ is the total number of neurons; $A_{x}$ is the weight value of neuron $x$; and $B_{x}$ is the output value of neuron $x$. The smaller the $P(x)$, the closer the distance between $A_{x}$ and $B_{x}$, and the more similar the two are.

In the use of this risk warning model, if there is too few hidden information points selected, the BP neural network will obtain too much effective information, which increases the training time. The number of best hidden information points can be obtained from the following formula:

$$
H_{x}=\frac{\sum_{x=1}^{n}\left(S_{x}-T_{x}\right)}{P(x)},
$$

where $H_{x}$ is the number of best hidden information points; the best training time can be obtained through calculation, and its efficiency $S_{x}$ and accuracy $T_{x}$ will be in the best state.

From the output layer to the hidden layer, the weights of the network are adjusted in the direction of reducing errors according to certain principles. In order to improve the connection weight along the gradient of $e$, the network gradually converges, and the BP neural network sets the weight $\alpha_{e}$ as

$$
\alpha_{e}=\left[P_{e}(\Delta x)-H_{e}(\Delta x)\right] \cdot f(e),
$$

where $f(e)$ is the connection weight between two nodes; $P_{e}$ is the connection weight along the gradient of $e ; \Delta x$ is the weight change. The neuron model is composed of neurons, firing functions, and connection weights between neurons. Each neuron receives information transmission from other neurons, and the total input connection weight $W_{i j}$ is

$$
W_{i j}=\int_{i=1}^{n}\left[k_{i j} x_{i}+\alpha_{e}(i)\right]
$$

where $k_{i j}$ is the binding strength of neuron $i$ and neuron $j$, called the connection weight; $x_{i}$ is the output of neuron $i$, and $\alpha_{e}$ (i) represents the threshold of neuron $i$.

The training samples modify the value of $i$ according to the error according to a certain method, so that the network can continue to develop in the direction of correct response, and the difference between the output of the network and the expected output is within the allowable range. Generally, the error square sum $E$ of the network output is the smallest, as the ideal result of network learning; $E$ is also called the energy function:

$$
E=\frac{1}{u} \cdot \sum_{i=1}^{n} \sum_{j=1}^{m} r\left[W_{i j}-\overline{W_{i j}}\right]^{2},
$$

where $u$ is the number of neurons in the hidden layer, $m$ is the number of neurons in the input layer, $n$ is the number of 
neurons in the output layer, and $r$ is a constant randomly selected from 1 to 10 . The method to determine the actual number of neurons in the hidden layer is to first calculate the general range of the number of neurons through the above formula and then select the intermediate value as the optimal number of neurons.

2.2. Learning and Training of the BP Neural Network. The processes extract a sample $x_{i}$ from the sample set; $k_{i}$ is set to be the threshold value from the input layer node to the hidden layer, and then, the output value $y_{i}$ of $i^{\text {th }}$ neuron in the hidden layer is

$$
y_{i}=\int_{i=1}^{n} k_{i}\left(l d_{i}-b u_{i}\right)
$$

where $d_{i}$ is the connection weight of each node in the input layer to each node in the hidden layer; $u_{i}$ is the connection weight of each node in the hidden layer to each node in the output layer; $l$ is the expected output value; $b$ is the adjustment and error value of the weight.

The BP neural network usually selects the typical forward feedback network error performance function to evaluate the performance of network $U_{i j}$, and its expression is

$$
U_{i j}=\frac{1}{m n} \sum_{i=1}^{m} \sum_{j=1}^{n}\left(\rho_{i j}-\sigma_{i j}\right)^{2},
$$

where $m$ is the number of training samples; $n$ is the number of neurons in the output layer; $\rho$ is the expected output; $\sigma$ is the actual output.

The algorithm continuously corrects the weights based on the error between the actual output value of the neuron and the expected output value [10]. The weight adjustments of the hidden layer and output layer neurons in the selflearning training model are

$$
y_{i j}=\alpha U_{i j}+\beta W_{i j}+\gamma E_{i j}
$$

where $y_{i j}$ is the weight of the input layer neuron $i$ and the rule layer neuron $j$ :

$$
Y_{i j}=1+c\left(\frac{y_{i j}}{\alpha_{i j}}-\frac{y_{i j}}{\beta_{i j}}-\frac{y_{i j}}{\gamma_{i j}}\right)^{2},
$$

where $\alpha_{i j}$ and $\beta_{i j}$ are the evaluation scores of the positive index and the inverse index, respectively; $\gamma_{i j}$ is the actual value of $i^{\text {th }}$ index; and $c$ is the standard value of $i^{\text {th }}$ index.

Through a certain number of the network training process, it is actually to modify the network parameters to determine the appropriate weights so that the input of all $n$ samples, the residual difference between the actual output $o$ and the expected output $o$, is minimized:

$$
Q_{t}=\frac{1}{2} \sum_{i, j=1}^{n} \frac{Y_{i j}}{t} .
$$

The correction of weights and control parameters is realized by the gradient method of the back propagation method; $t$ is the number of iterative corrections; and $Q_{t}$ is the control parameters in the fuzzy operator of the rule layer and the output layer, respectively.

\section{Construction of the Early Warning Model of Human Resource Management Risk Based on the BP Neural Network}

\subsection{Risk Inducement Analysis and Index System Establishment} of Human Resource Management. The BP neural network not only can qualitatively analyze methods to be used for human resource risk early warning but through the introduction of the previous BP neural network, it is understood that it can also quantitatively analyze the human resource risk early warning of an enterprise. Through field research on the company, it can get the completion rate of employees' work goals, the rate of employee training excellence, the ratio of internal and external personnel, employee satisfaction, employee salary growth rate, employee benefits improvement rate, new employee growth rate, employee promotion rate, and employees human resource risk early warning indicator data for 10 samples of turnover rate and employee attendance rate and input these data as samples into the BP neural network model. After the analysis of the BP neural network, the output data are graded by the expert scoring method, and the grade is determined according to the amount of data. After the data are processed in a consistent manner, it is found that the security level and data of each enterprise are obtained after the use of the BP neural network risk warning meets the requirements, indicating that the evaluation system can be used in actual human resource risk warning. Human resource risk is a key link that cannot be underestimated in the operation of an enterprise, which is one of the most valuable resources of an enterprise and the backbone of the company's future development.

The basic process of the early warning model, the first step, is to do a principal component analysis of the indicators in the enterprise marketing risk early warning indicator system, and the principal components obtained are used as the input of the BP neural network, and the value representing the corresponding comprehensive evaluation result is used as the neural network output. The second step is to train the network on the sample set of the existing evaluation results, and the third step, testing, is to use the test samples in the sample set to test the validity of the model. The set of weights and thresholds held by the neural network is the correct internal representation obtained by the network through white adaptive learning to the mapping between evaluation values. Although the early warning indicator system established above can fully reflect the various internal and external risks faced by the enterprise, the number of indicators is too large, and there may be a certain correlation between these indicators, and too many indicator values are directly used as the neural network. Inputting variables will complicate the network structure, increase the training burden, and slow down the learning rate, which will affect the difficulty of evaluating model training (Figure 1). Therefore, it is necessary to extract these indicators and find the factors that can most 


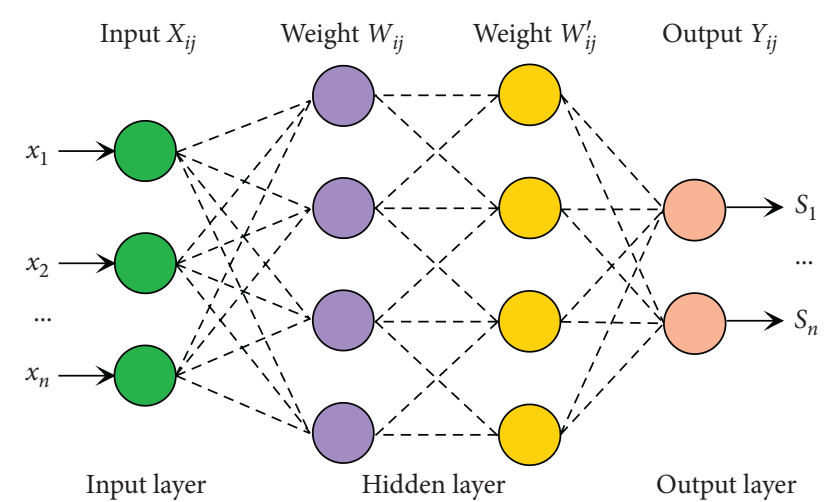

Figure 1: Structure distribution of the BP neural network model.

influence the evaluation value of informatization as the input of the neural network [11].

In enterprise human resource management, risk early warning indicators refer to the formation of indicators that can accurately reflect the status of human resources management activities and the results of investigations and are suitable for the investigation of certain fixed indicators that are difficult to collect specific values such as job matching and comprehensiveness of assessment based on the BP neural network, building an early warning model of human resource management risk that can more intuitively and accurately make risk early warnings for enterprises [12]. The principal component analysis method is mainly used to preprocess the simulation data, and the larger the index system, the greater the risk of human resource management. In the selection of input nodes of the BP network model, the BP neural network processes the original simulation data in a unified manner, calculates the largest sum of square deviations before the evaluation object as much as possible, and predicts that the enterprise human resource management risk is in a large or small state. The choice of hidden layer nodes in the BP network model should be based on the actual situation of the network because if the number of hidden layer nodes is too small, the network may not be trained or the network cannot recognize samples that have not been stored before, so the actual output and the expected output vector. The size of the mean square error and the number of training steps are comprehensively analyzed and the appropriate number of hidden layer nodes is determined, which has reached the optimization of human resource management risk early warning.

3.2. Node Selection of the BP Neural Network. The artificial neural network has learning ability, and the BP neural network has good input-output mapping ability through learning. When this article uses the BP neural network early warning model for empirical research, the main purpose of the selected representative data under various risk states is to reflect the overall risk status of the channel, and the BP neural network is trained to make each neuron hidden. Containing ground is affected by the global activities of other neurons, so neural networks can naturally dig out valuable knowledge and laws from large amounts of data. Inductive ability means that the BP neural network can generate reasonable output for new inputs during the training process, which greatly reduces the difficulty of risk assessment of corporate marketing channels [13]. During the signal forwarding process, the weights and thresholds of the network are fixed, and the state of each layer of neurons only affects the state of the next layer of neurons. In the early warning process, it is inevitable that there will be local indicator variation, which will form early warning noise. However, due to the highly parallel distribution of information storage in artificial neural networks, the local damage will weaken the operation of artificial nerves. There will be no overall impact, and the alarm can be accurately prewarned based on existing samples.

The index system is divided into three levels: the first level is the general index system; the second level is the main index system, which is composed of four types of causes of operational risk; the third level is the detailed index system, which is the detailed factor affecting the four types of causes. The acquisition of detailed index system data must be based on the information data system of human resources. A large amount of time sequence factor data are analyzed and calculated through the BP neural network model, and the neural network prediction model is obtained, so as to make the future risk status and correct early warning judgment. Due to the many factors involved in operational risks, some of the influencing factors are not easy to obtain from the human resource system. Manual statistical calculations are required afterward, which affect the accuracy, effectiveness, and real-time of the early warning system to a certain extent [14]. When inputting data, it is necessary to process all data according to the principle of variable normalization to make it a dimensionless index value on a closed interval (Figure 2). In order to establish an effective, scientific, and real-time operational risk early warning system, the personnel department should complete the construction of the indicator information system as much as possible. In addition to the calculation factors in the key risk indicators, other quantitative factors that may form operational risks should also be included. The scientific operational risk early warning system will provide extremely effective means and help for human resource management to prevent it.

Under the guidance of organizational vision and organizational goals, human resource management grasps the demand and supply of human resources from a strategic height according to the characteristics of human resource activities, dynamically plans human resources, and strives to balance human resource demand and supply, thereby promoting organizational goals achieve. The node selection of the BP neural network has the following characteristics: the organization of human resource management activities is highly compatible with the business strategy; the organization's horizontal and vertical organization settings match the needs of internal and external human resource management behaviors; employees at all levels within the 


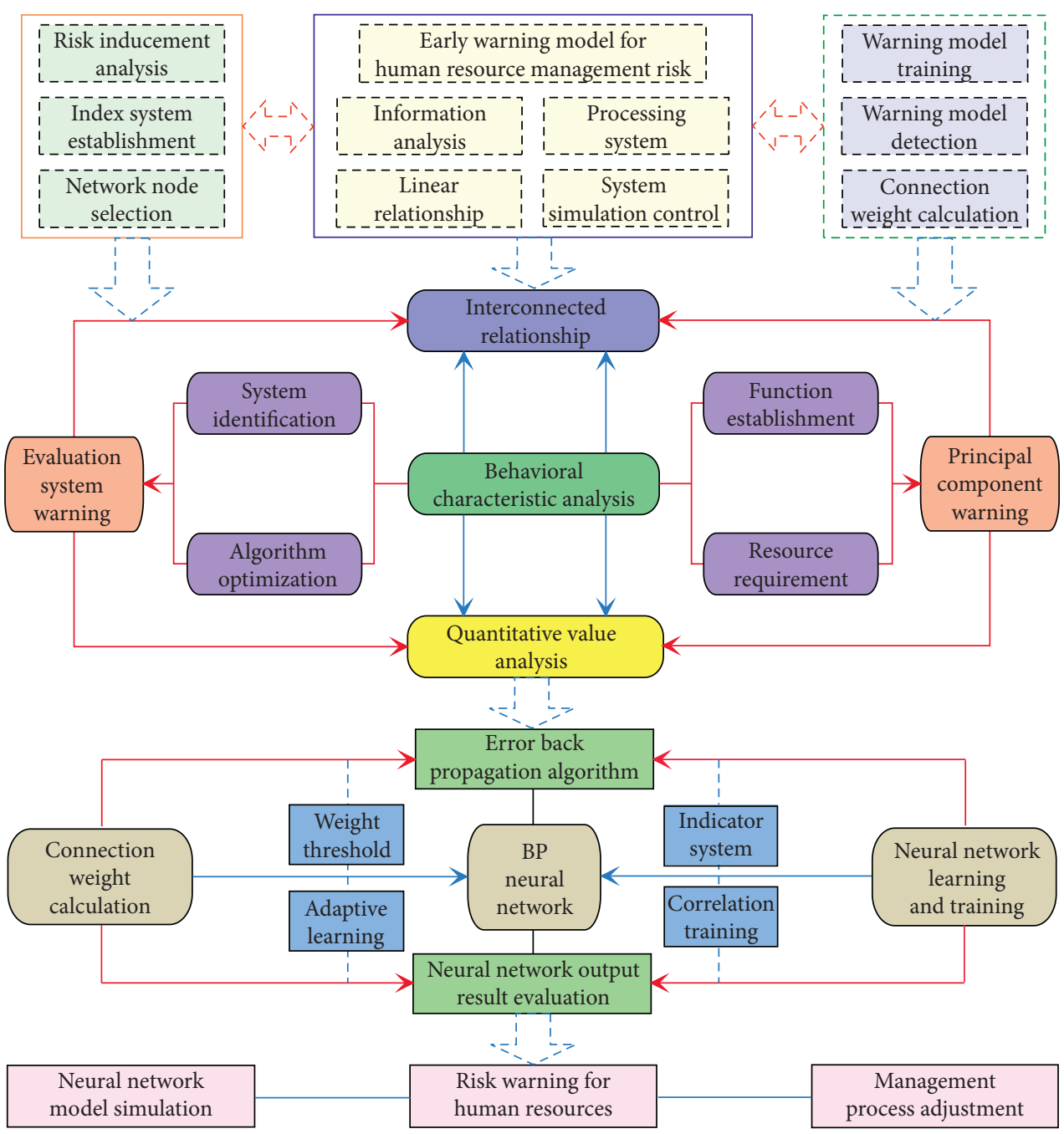

FIGURE 2: Analysis and simulation framework of the early warning model for human resource management risk based on the BP neural network.

organization carry out practices based on human resource management standards and stay dynamic with organizational strategy. However, both the explicit elements and the hidden elements are important references for the evaluation and selection of talents of different job categories and levels. As an important foundation for the realization of strategic human resource management goals, talent evaluation helps organizations build a diversified talent team that meets their development vision. It plays a key role in stabilizing the foundation of organizational development, improving the development potential of organizational talents, eliminating low-performing personnel who are not compatible with organizational development strategies, and innovating organizational development vitality.

\section{Training and Detection of the Risk Warning Model Based on the BP Neural Network}

4.1. Risk Warning Model Training. The selection of hidden layer nodes is a very complicated problem because the massive parallel distributed structure and nonlinear dynamic characteristics of neural networks determine that a simple and universal concise analytical expression or hidden layer unit determination formula is very theoretically obtained. The choice of the number of hidden layer nodes is directly related to the requirements of the problem and the number of input and output units: if the number of hidden nodes is too small and the network is not strong, it will not be able to identify samples that have not been seen before, and the fault tolerance will be poor. Too small structures may not be trained, but too many hidden nodes will make the learning time too long, and the error may not be the best (Figure 3). The BP neural network can proceed from the overall situation, adjust specific data, and accurately turn on the alarm and alarm between warning indicators and warning levels. It can see the process of optimizing human resource management risk early warning and gradually approach a function. In the risk of human resource management, when a risky factor is constantly developing, it is likely to threaten the human resource management of the entire enterprise. The BP neural network has global characteristics and can accurately help the human resource 


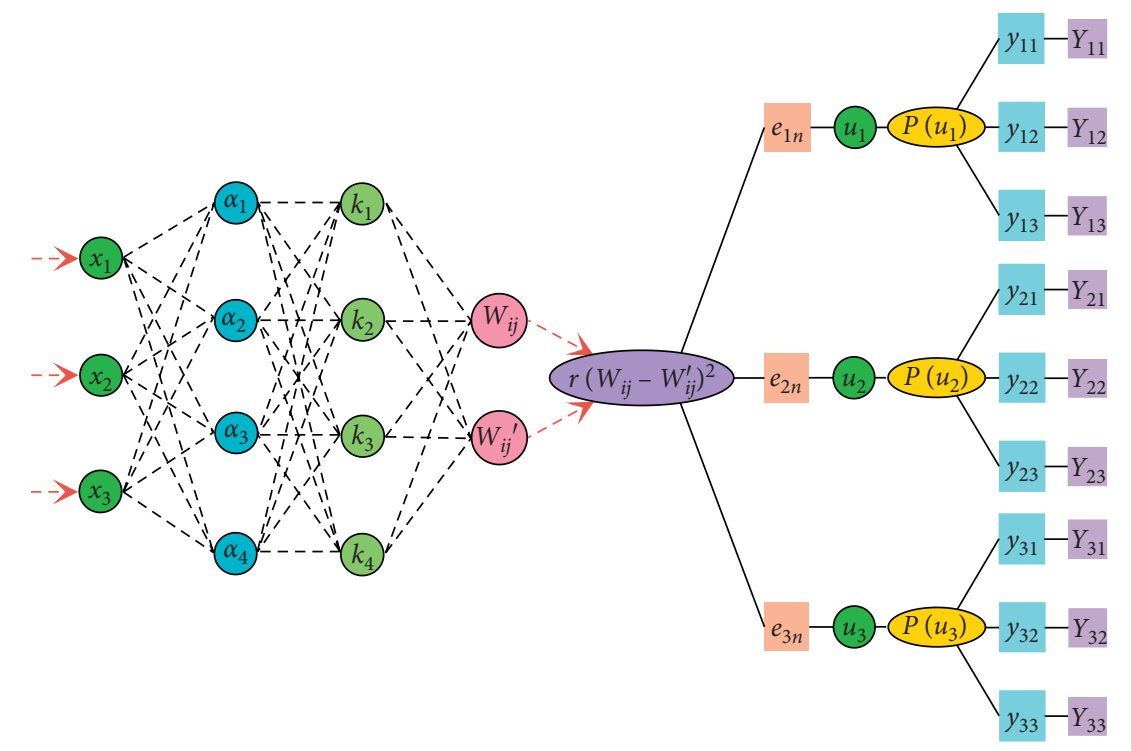

FIGURE 3: Analysis process of the human resource management risk inducement system based on the BP neural network.

management of the enterprise. Early warning is made when there is a risk, which is extremely applicable [15].

In order to avoid the increase in the number of hidden layers, the network learning and training time will increase and the error value will increase. This study chooses one layer as the number of hidden layers, and it can also increase the number of neurons on the basis of a hidden layer. To improve the accuracy of BP neural network training, in terms of structural realization, this is simpler than increasing the number of hidden layers. Of course, the number of neurons in the hidden layer of a neural network is not as large as possible, and the principle must be selected. Generally speaking, the greater the number of nodes in the hidden layers of the network, the greater the function. However, the advantage is that the performance is stronger, but the disadvantage is that the structure is complicated and the number of cycles, that is, the training time, increases. The learning and training efficiency becomes low, and the error value control cannot reach the best state, and the number of neurons is too small; its advantage is that the learning and training time is less and the network structure is simple and the disadvantage is that the performance ability is not strong and the learning ability and the information processing ability are poor. The fault tolerance is poor, and the learning error declines slowly and even nonconvergence occurs. However, due to the parallel distribution of the BP neural network structure and the existence of nonlinear dynamics, it is difficult to unify the formula for determining the number of neurons in the hidden layer.

The unique nonlinear adaptive information processing ability of the artificial neural network overcomes the shortcomings of traditional artificial intelligence methods in pattern, speech recognition, and unstructured information processing, so that it can be used in function approximation, pattern recognition, and data compression. Since the statistical method does not require premises and assumptions, it only needs to substitute the values of various indicators into the model for regression analysis to draw conclusions. Therefore, it is not too complicated to operate, but its shortcomings are also obvious, and more and more successful examples have emerged with increasing advantages. Because a single index system cannot contain or reflect all the information about resource management risks, it is also unconvincing to judge the management risk status based on a single index system [16]. If multiple indicators are used at the same time to evaluate the risks of human resource management separately, the prediction results of these indicators may not be completely inconsistent, which prevent decision makers to make correct judgments and take corresponding countermeasures. Despite the above shortcomings, the statistical method has made researchers realize that the risk status of management is the result of the superposition of multiple risk factors, which has a very important guiding significance for the ideological enlightenment of early warning model research and technological progress and influence.

4.2. Risk Warning Model Detection. The principle of human resource management risk early warning evaluation based on the BP neural network is to take the secondary indicators of human resource management risk as the input of the neural network, take the comprehensive evaluation of knowledge management risk as the output, and use enough samples to train the network and calculate the weights and thresholds. After the neural network learning and training is completed, the model can be used to make corresponding comprehensive evaluation of management risk early warning for different human resources. Under normal circumstances, increasing the number of hidden layer neurons can reduce network errors and improve accuracy, but it will also complicate the network, thereby increasing the network training time and increasing the possibility of overfitting (Figure 4). Data preparation is to extract certain 
training samples from the obtained human resource management risk early warning secondary indicator data and input the standardized secondary indicator data into the network. In the construction of human resources, project managers often introduce talents and increase the research and development of new processes to achieve high-performance human resources knowledge sharing risks in project construction. The risks are not only caused by the efficiency of knowledge transfer and communication between employees within the project, which may also be caused by management spill-over effects caused by knowledge exchange and sharing with relevant organizations or units outside the project.

Human resource management risk indicators should be able to sensitively reflect the status of human resource management activities and potential risk factors in talent management work. Through the analysis of the actual status of human resource management in the county, combined with the questionnaire survey content, the risk of human resource management is divided into five categories, namely, talent training, talent use, talent flow, talent distribution, and talent structure, and then according to the principles of comprehensiveness, difference, comparability, and scientific, five specific risk indicators are obtained after screening. When there are too many hidden layer nodes, the advantage is strong performance ability, but the disadvantage is that the training efficiency is not high, the generalization ability is reduced, and the best error may not be achieved; when there are too few hidden layer nodes, the advantage is that the learning time is less, and the disadvantage is the lack of performance power, fault tolerance is poor, and learning error decreases or even does not converge [17]. Model training can solve the hidden layer node number, weight, and threshold adjustment problems, so that the BP neural network can better meet the needs of real problems. According to the final output result of the operation test of the final data, compare and analyze its specific expected output with the actual output, and it can be known that the actual output of the constructed model data is basically consistent with the expected output, and the mean square error is within the acceptable error range.

The enterprise needs to determine a series of factors that may constitute the enterprise's human resource management risk for evaluation. It is good to be able to give a unified risk factor for analysis, but in fact, the risk factors that different companies pay attention to are often different, which require that the possible risks must be identified first. In the previous article, a number of human resource management risk factors have been given as evaluation indicators, and companies can choose them according to actual conditions. For each risk factor, due to differences in knowledge, experience, and values, different evaluators have different perceptions of the possible impact of each risk factor. The risk evaluation of enterprise human resource management has obvious fuzzy characteristics; so, the theories and methods used in the establishment of evaluation and early warning models should adapt to this characteristic. Because of this characteristic, the evaluation of the severity of risk cannot be analyzed and judged from the enterprise's own mechanism, which is necessary to set up a risk evaluation team composed of middle and high-level leaders and experts of the enterprise to score. Different companies pay very different attention to risk factors, and this requires a judgment based on the actual situation of the company on the severity of the risk that may affect the company [18].

\section{Simulation and Result Analysis}

5.1. Case Company and Sample Data. In the operation of the model, the following parameters are selected: the learning rate is 0.05 , the system error is 0.001 , the number of runs is 20000 times, and the corresponding function is selected as the activation function. For the number of hidden layer nodes, based on the reference formula, first determine the smaller number of hidden layer nodes for training. If there is no convergence within the specified number of training times, it will stop training and gradually increase the number of hidden layer nodes. This study chooses four cases, namely, $6,10,12$, and 14 . After multiple training, the number of hidden layer nodes is finally determined to be 20 , so the BP network model structure of this article is $8 \times 10 \times 1$. Through field research on the company, the system can get the completion rate of employees' work goals, the rate of employee training excellence, the ratio of internal and external personnel, employee satisfaction, employee salary growth rate, employee benefits improvement rate, new employee growth rate, employee promotion rate, and employees human resource risk early warning indicator data for 10 samples of turnover rate and employee attendance rate. The analysis and simulation workflow of the early warning model for human resource management risk based on the BP neural network is shown in Figure 5.

The research object of the early warning model established in this paper is human resource management risk, and the input layer of the model should be human resource management risk early warning inducement index data. Therefore, this paper selects multiple risk indicators as input nodes. Because these indicators have different measurement standards, both quantitative and qualitative, their nature and dimensions are also different, which makes it impossible to measure the indicators together [19]. Therefore, in order to better input data and smoothly connect research objects and models and speed up the convergence of the learning and training networks, before inputting data, this article first normalizes all sample data and converts them into closed interval nondimensional index values, thereby eliminating the problem of ambiguous warning results caused by different index properties and nonsuperposition.

5.2. Result Analysis. The basic idea of using the BP neural network to carry out risk evaluation is that it is often necessary to organize a large number of experts to ensure the effect of the evaluation when carrying out risk evaluation on investment projects, but in actual operation, this is often impossible. Therefore, the BP neural network can use authoritative evaluation results as the input and output of the 


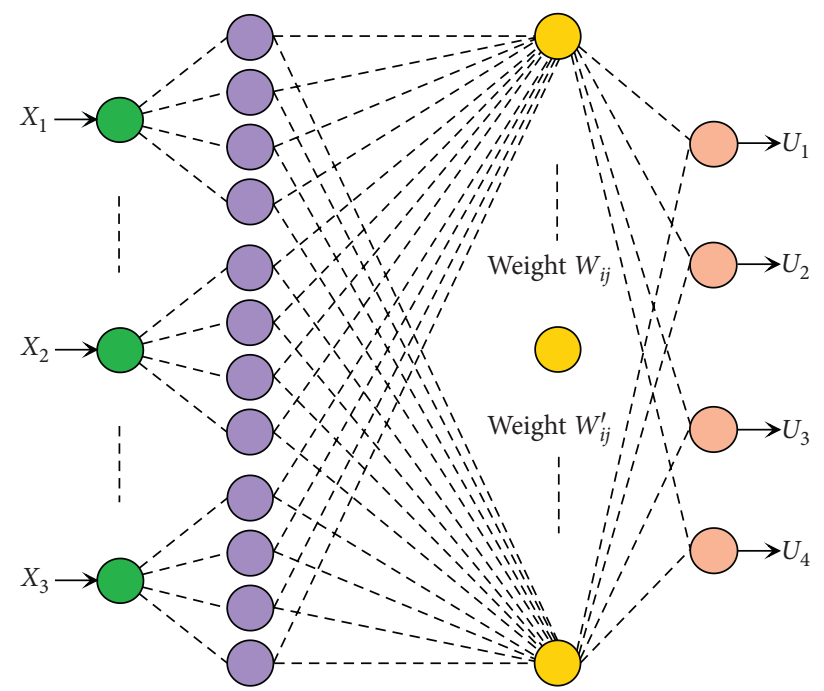

FIGURE 4: BP neural network model of human resource management risk.

neural network and train the network until the training accuracy of the neural network reaches the established requirements [20]. The trained network only needs experts to give the index value of each risk for a certain project, and the neural network can give a comprehensive evaluation of the investment risk of the project. In this way, when it needs to evaluate the investment risk of the investment object, it can take out the trained network, invite experts to analyze the weight of the project to be evaluated, calculate the score, and substitute it into the trained neural network, and the neural network can output the corresponding, and the risk level of the project is obtained (Figure 6). The multiobjective comprehensive evaluation method based on the BP neural network, through the self-learning, self-organizing adaptability, and strong fault tolerance of the neural network, can accurately work according to the expert evaluation method. The trained neural network system is to connect the expert evaluation thoughts.

The purpose of human resource management is to achieve the purpose of human integration and integration through the full and extensive sharing of various basic or professional human resources and to carry out human innovation and rapid product innovation on this basis. Insufficient human resources will result in low innovation effects and efficiency, leading to the failure of innovation or the commercialization of innovation results, and ultimately damage the economic benefits of enterprises. The diagnostic accuracy of this algorithm mainly depends on the number and quality of the cut-to-I training samples, but it is difficult to obtain enough ideal samples at a time in practice. This needs to be accumulated gradually during use, and the network tends to be perfected through continuous learning. The selection of the number of hidden layer nodes in the network is a complicated problem. Too few hidden layer nodes will cause too many local minimums, and too many nodes will make the learning time too long and the error may not be the best. The BP algorithm is composed of two parts: the forward transmission of information and the back propagation of errors. In the forward transmission process, the input information is passed from the input layer to the output layer through the hidden layer calculation. If the desired output is not obtained in the output layer, then calculate the error change value of the output layer, turn to back propagation, and pass the error signal back along the original connection path through the network to modify the weight of each layer of neurons until the desired goal is reached [21].

The BP neural network can proceed from the overall situation, adjust specific data, and accurately turn on the alarm and alarm between warning indicators and warning levels. In this study, the warning levels are divided into four different levels (I-IV) to describe the training and detection results of the risk warning model based on the BP neural network. The artificial neural network does not need to determine the mathematical equation of the mapping relationship between the input and output in advance. The early warning model of human resource management risk based on the BP network is formatted, and its core process includes four aspects, namely, function approximation, pattern recognition, classification, and data compression (Figure 7). In the actual human resource risk management of an enterprise, this function can be regarded as various situations that cause risks, such as insufficient human resource management funds and brain drain [22]. The BP network early warning model of human resource management risk applies this principle to work, and its basic requirements include three aspects, namely, adequate understanding of the work content, smooth, fast, and reasonable disposal of information in and out of channels. When there are human resource management risks, such as insufficient funds, the subresponsible person and their assistants will feedback the information to the general manager as soon as possible, and 


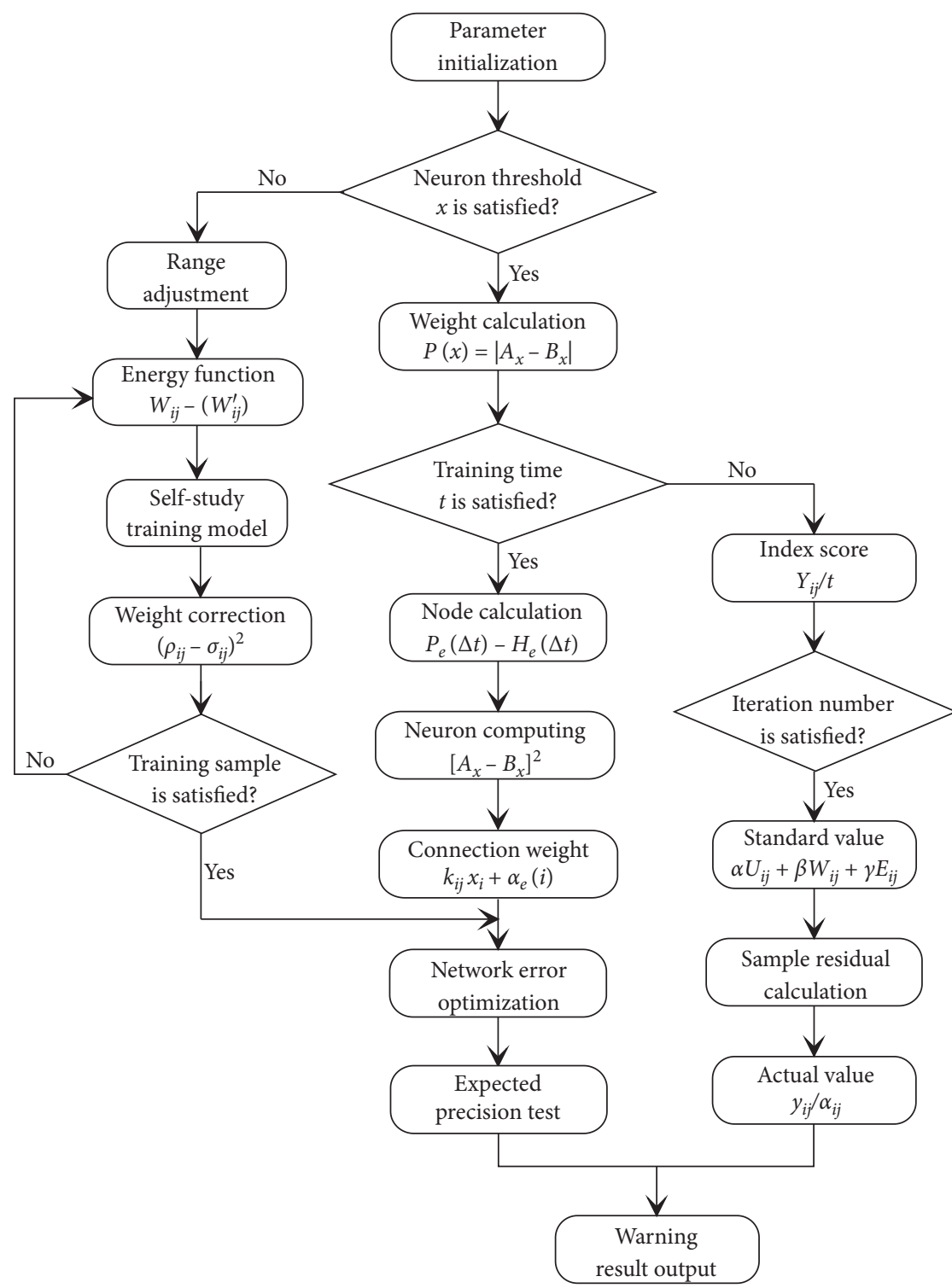

FIgURE 5: Analysis and simulation workflow of the early warning model for human resource management risk based on the BP neural network.

the general manager will negotiate with the senior management of the company to avoid the spread of risks and expand.

\section{Conclusion and Future Scope}

This paper performed the risk inducement analysis, index system establishment, and network node selection of human resource management, constructed an early warning model of human resource management risk based on the BP neural network, conducted the risk warning model training and detection based on the BP neural network, and finally carried out a simulation and its result analysis. The principle of human resource management risk early warning evaluation based on the BP neural network is to take the secondary indicators of human resource management risk as the input of the neural network and take the comprehensive evaluation of knowledge management risk as the output. For each risk factor, different evaluators have different perceptions of the possible impact of each risk factor due to differences in knowledge, experience, and values. The trained network only needs experts to give the index value of each risk for a certain project, and the neural network can give a comprehensive evaluation of the investment risk of the project. The early warning model of human resource management risk based on the BP network is formulated, and its core process includes four aspects, namely, function approximation, pattern recognition, classification, and data compression. The study results show that the early warning model of human resource management risk based on the BP network is effective, and this trained and tested BP network risk warning model can be used to conduct early warning empirical research on human resource risks to prevent human resource risks and ensure enterprise's benign 


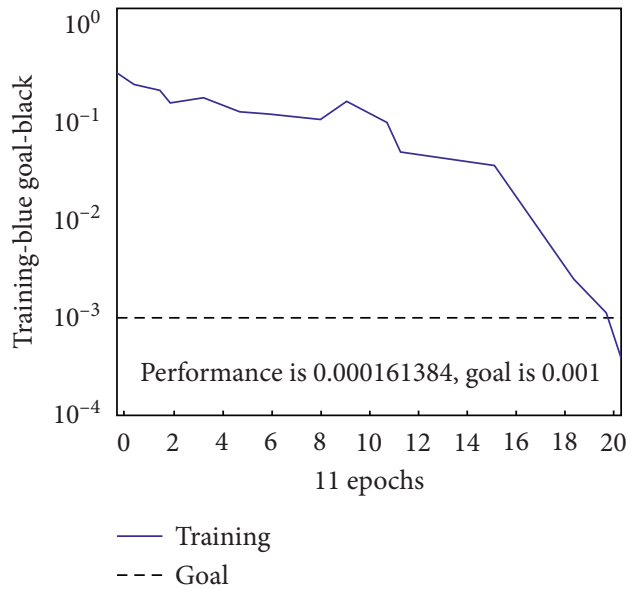

(a)

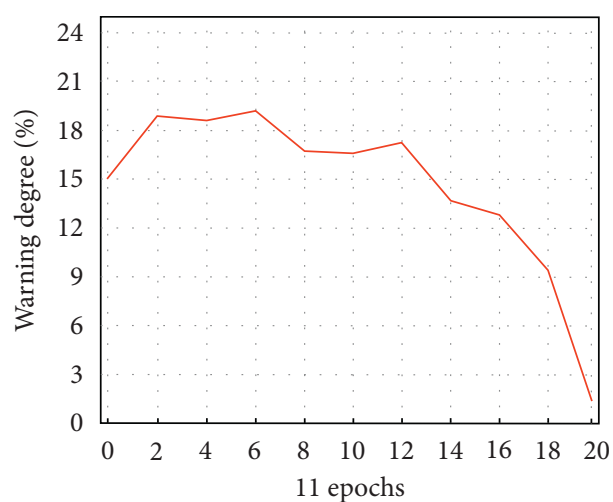

(b)

FIGURE 6: Training convergence (a) and training warning indicator (b) of the BP neutral network.

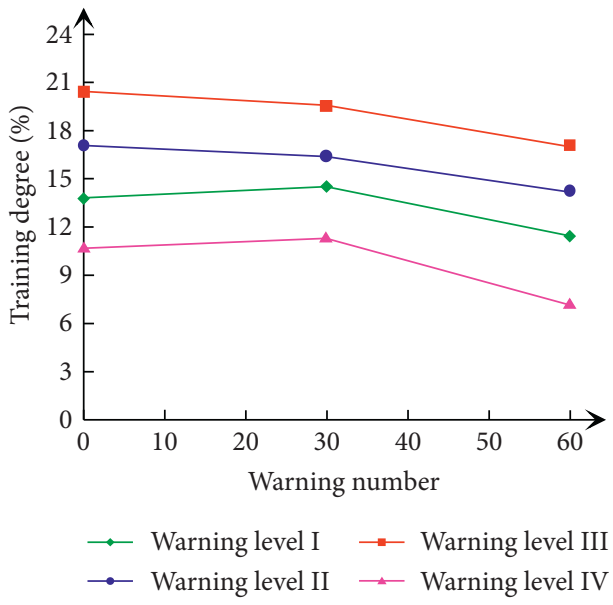

(a)

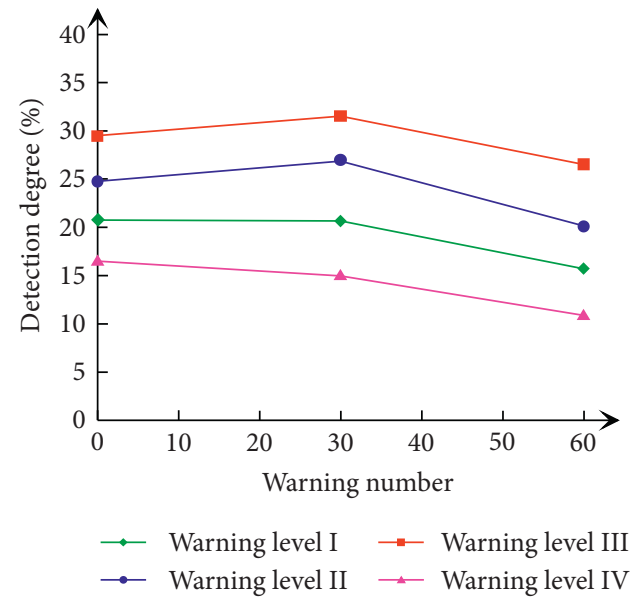

(b)

FIgURE 7: Training (a) and detection (b) results of the risk warning model based on the BP neural network.

operation and play a role in supervision and promotion of market order rectification. The study results of this paper will provide a reference for further research studies on the analysis and simulation of the early warning model of human resource management risk based on the BP neural network.

\section{Data Availability}

The data used to support the findings of this study are available from the corresponding author upon request.

\section{Conflicts of Interest}

The authors declare that they have no conflicts of interest.

\section{References}

[1] P. C. Patel, M. Li, M. d. C. Triana, and H. D. Park, "Pay dispersion among the top management team and outside directors: its impact on firm risk and firm performance," Human Resource Management, vol. 57, no. 1, pp. 177-192, 2018.

[2] M. S. U. Rajib and L. Fan, "A study on the critical factors of human error in civil aviation: an early warning management perspective in Bangladesh," Management Science Letters, vol. 5, no. 1, pp. 21-28, 2015.

[3] S. Park, S. Song, and S. Lee, "How do investments in human resource management practices affect firm-specific risk in the restaurant industry?" Cornell Hospitality Quarterly, vol. 58, no. 4, pp. 374-386, 2017.

[4] B. Bader, T. Schuster, and M. Dickmann, "Special issue of international journal of human resource management: danger 
and risk as challenges for HRM: how to manage people in hostile environments," The International Journal of Human Resource Management, vol. 26, no. 11, pp. 1517-1519, 2015.

[5] Z. Zhao, Q. Xu, and M. Jia, "Improved shuffled frog leaping algorithm-based BP neural network and its application in bearing early fault diagnosis," Neural Computing and Applications, vol. 27, no. 2, pp. 375-385, 2016.

[6] W. Jia, D. Zhao, T. Shen, S. Ding, Y. Zhao, and C. Hu, "An optimized classification algorithm by BP neural network based on PLS and HCA," Applied Intelligence, vol. 43, no. 1, pp. 176-191, 2015.

[7] D.-Z. Huang, R.-X. Gong, and S. Gong, "Prediction of wind power by chaos and BP artificial neural networks approach based on genetic algorithm," Journal of Electrical Engineering and Technology, vol. 10, no. 1, pp. 41-46, 2015.

[8] C. Qiu and J. Shan, "Research on intrusion detection algorithm based on BP neural network," International Journal of Security and Its Applications, vol. 9, no. 4, pp. 247-258, 2015.

[9] M. Mackay and A. Tymon, "Taking a risk to develop reflective skills in business practitioners," Journal of Education and Work, vol. 29, no. 3, pp. 332-351, 2016.

[10] W. Li and L. Ren, "Research on the risk of human resource management outsourcing for first-class hotel --- a case of InterContinental Shenzhen," Universal Journal of Management, vol. 4, no. 6, pp. 361-366, 2016.

[11] Z. M. Talib, E. Kiguli-Malwadde, H. Wohltjen et al., "Transforming health professions' education through incountry collaboration: examining the consortia among African medical schools catalyzed by the medical education partnership initiative," Human Resources for Health, vol. 13, no. 1, pp. 1-8, 2015.

[12] D. E. Guest, "Human resource management and employee well-being: towards a new analytic framework," Human Resource Management Journal, vol. 27, no. 1, pp. 22-38, 2017.

[13] D. L. Stone, D. L. Deadrick, K. M. Lukaszewski, and R. Johnson, "The influence of technology on the future of human resource management," Human Resource Management Review, vol. 25, no. 2, pp. 216-231, 2015.

[14] O. Klopova, L. Komyshova, and M. Simonova, "Professional development in the field of human resource management of heads and specialists of the innovative organizations," Problems and Perspectives in Management, vol. 16, no. 1, pp. 214-223, 2018.

[15] T. Pritvorova, B. Tasbulatova, and E. Petrenko, "Possibilities of Blitz-Psychograms as a tool for human resource management in the supporting system of hardiness of company," Entrepreneurship and Sustainability Issues, vol. 6, no. 2, pp. 840-853, 2018.

[16] F. Hu, L. Wang, S. Wang, X. Liu, and G. He, "A human body posture recognition algorithm based on BP neural network for wireless body area networks," China Communications, vol. 13, no. 8, pp. 198-208, 2016.

[17] Y. Zhang, X. Gao, and S. Katayama, "Weld appearance prediction with BP neural network improved by genetic algorithm during disk laser welding," Journal of Manufacturing Systems, vol. 34, pp. 53-59, 2015.

[18] B. Wu, S. Han, J. Xiao, X. Hu, and J. Fan, "Error compensation based on BP neural network for airborne laser ranging," Optik, vol. 127, no. 8, pp. 4083-4088, 2016.

[19] H. Jia, D. Pan, Y. Yuan, and W. Zhang, "Using a BP neural network for rapid assessment of populations with difficulties accessing drinking water because of drought," Human and Ecological Risk Assessment: An International Journal, vol. 21, no. 1, pp. 100-116, 2015.
[20] X. Han, X. Xiong, and F. Duan, "A new method for image segmentation based on BP neural network and gravitational search algorithm enhanced by cat chaotic mapping," Applied Intelligence, vol. 43, no. 4, pp. 855-873, 2015.

[21] S. Liu, Z. Hou, and C. Yin, "Data-driven modeling for UGI gasification processes via an enhanced genetic BP neural network with link switches," IEEE Transactions on Neural Networks and Learning Systems, vol. 27, no. 12, pp. 2718-2729, 2016.

[22] K. Zhang, F. Yuan, J. Guo, and G. Wang, "A novel neural network approach to transformer fault diagnosis based on momentum-embedded BP neural network optimized by genetic algorithm and fuzzy c-means," Arabian Journal for Science and Engineering, vol. 41, no. 9, pp. 3451-3461, 2016. 